\title{
Modeling and Simulation of an Autonomous Power Manager for Sensor Networks
}

\author{
Rym Che'our, Mohamed Wassim Jmal, and Mohamed Abid
}

\begin{abstract}
Wireless Sensor Networks (WSN) have widespread applications, ranging from the industrial automation to the home applications, a result of their higher enhanced capabilities and im-proved cost-efficiency. However, there may not be enough resources to process those applications. So raising the energy efficiency of WSN is a paramount objective to extend the lifetime of the sensors network. This paper presents the methodologies followed to develop effective techniques to save the energy consumption of the WSN at the system level and under time constraints. This approach is based on an interplay of online strategies to manage the energy at a local level, as well as a scheduling policy that exploits effectively the resources at the global level. The autonomous power manager combines the Dynamic Power Management (DPM) with a Dynamic Voltage and Frequency Scaling (DVFS) that will be validated using the STORM simulator.
\end{abstract}

Index Terms-Wireless sensor network (WSN), energy efficiency, DVFS, DPM, scheduling, modeling.

\section{INTRODUCTION}

The wireless sensor networks are made up of many nodes placed without any control during months or years. Each node is designed to handle its energy supply in order to maximize the total lifetime of the network [1]. Besides, a sensor can exhaust its energy completely and become nonoperational because of the expiry of its battery [2]. The lifetime of a sensor node depends primarily on two factors: the quantity of energy consumed in time, and how much energy is available for its use [3]. As a result, the WSNs require a global management of energy: at the level of the network the activity of the node should be controlled in order to maximize the lifetime of the battery while decreasing the energy consumption under time constraints [4]. We have noted that the synchronization of the tasks does not allow a comprehensive use of all the capacity of the CPU of a single node as there is no overall control. For this purpose, it is necessary to set up a local energy management at the node level. Indeed, the control of the development by scheduling helps to analyze the WSN model [5].

The concepts developed in our work assume that WSNs nodes do not have the possibility of energy harvesting. They are thus, conceived to optimize the lifetime of a node by optimizing their own energy consumption. In this paper, the application selection, the architecture and scheduling choices considered will be defined as well as the methodology to

Manuscript received February 20, 2016; revised May 21, 2016.

The authors are with the National School of Engineers of Sfax, Computer and Embedded System Laboratory, University of Sfax, Tunisia (e-mail: cheourr@gmail.com, wassim.jmal@gmail.com, Mohamed.Abid@enis.rnu.tn) jointly manage the real-time criteria and the low consumption. Unlike traditional energy management systems, we have relied on energy optimization techniques on the one hand, to yield extensive lifetime for every nodes battery and mainly both DPM(Dynamic Power Management) and DVFS(Dynamic Voltage and Frequency Scaling) that are appropriate for the WSN. On the other hand, this model will be based on a global EDF(Earliest Deadline First) scheduling policy and simulated with STORM [6]. At the local level, we will apply the DPM technique during the idle interval, and when the node is active the strategy DVFS will be used. To avoid missing deadlines and to provide schedulability guarantees, we will implement a G-EDF at the global level.

The architecture of the power manager model is made up of four basic components. The tasks allocation phase includes the specification of the software and hardware architecture defined in the XML file. Also, the task scheduling module prioritizes tasks and resource requests via task scheduling and mainly by the G-EDF scheduler. The energy management component tries to control the resource usage by selecting between DPM and DVFS. Finally, the power evaluation component aims to give a feedack about the performance criteria such as the energy gain or the respect of time constraints. The use of the simulation rather than hardware prototyping is marked by many advantages like a significant cost savings, compressed validation time, and improved component analysis [7], [8].

The reminder of the paper is organized as follows. In sectionII, we will give a brief summary of the related work. It includes an overview and a comparison of the existing techniques. In the next section, will describe the WSN power manager. Section IV will give some results. Finally, a conclusion and future work are given.

\section{RELATED WORK}

Many power saving mechanisms have been already incorporated into various standards and protocols [9]. Also, ultralow-power wireless sensor networks have been used to get power-efficient systems [10]. Some techniques, at the local level, require decisions at the global level and vis versa. So, when we consider the impact of both approaches alongside WSN consumption, a much greater saving is driven. This is explained in the next section which highlights the intrinsic relationship between them and describes with more details how the model will be developed on the actual plan. The need behind our work comes from the fact that no single policy suits perfectly all the operating conditions. So, we will try to combine several existing policies to have better results by taking into account the application requirements. 


\section{Simulators}

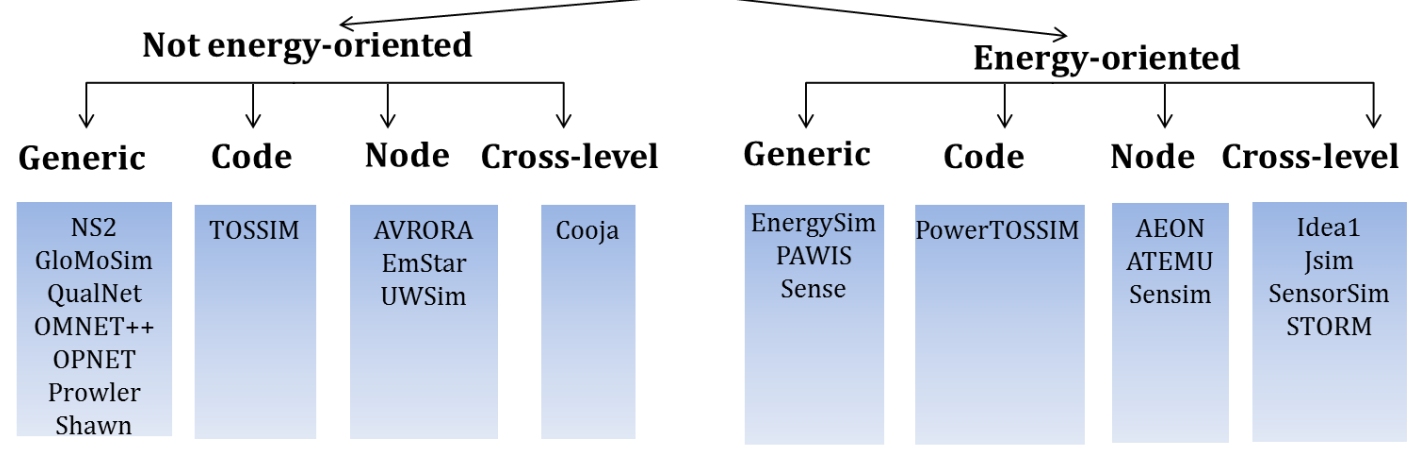

Fig. 1. Taxonomy of simulators for wireless sensor networks.

TABLE I: COMPARISON OF SCHEDULES

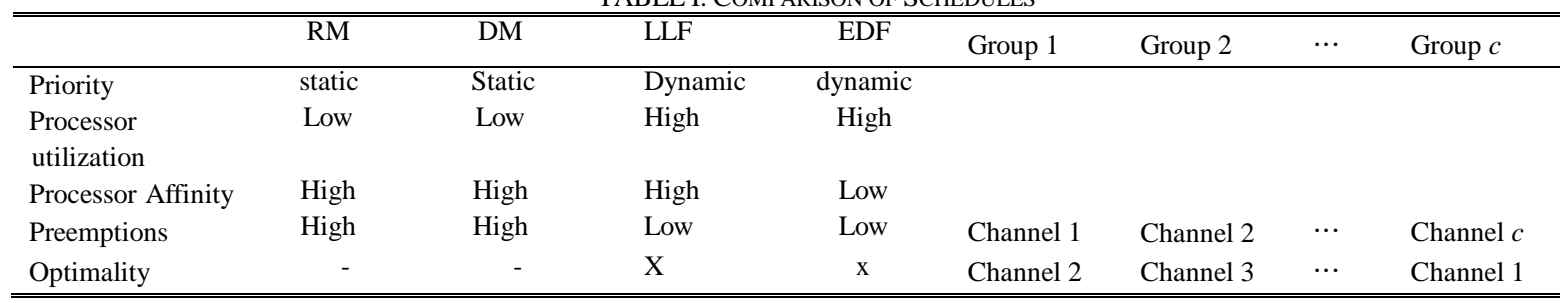

Indeed, the recent studies move towards the employment of energy harvesting techniques as a solution to improve the energy efficiency [11]. The energy-scavenged in particular that coming from intermittent and variable sources (e.g., implantable batteries and capacitive methods), poses additional challenges like the availability. Moreover, most of the methods are still under investigation and provide low energy savings. They may also cause hazards and skin infections when de- ployed in the healthcare monitoring [12]. The power profile of the task set can be varied by exploiting DVFS and DPM capabilities of the proessor. Only few publications address the use of both DPM and DVFS in WSN. In [13], authors present an implementation of DPM and DVFS techniques. But, these techniques, alone, are not sufficient to ensure a sustainable operation. However, not relying on scheduling can lead to significant loss of performance criteria. Indeed, non- compliance with time constraints may affect the efficacy of the network.

The scheduling policy is chosen according to its capacity to manage real-time tasks and to allow effective optimizations of energy. Several algorithms of scheduling are optimal in case single processor, like EDF (Earliest Deadline First) or LLF (Least Laxity First) for which the CPU ustlistion $100 \%$ is possible while guaranteeing the respect of the deadlines [14]. But, LLF has larger overhead than EDF due to higher number of context switches caused by laxity changes at run time. Besides, these algorithms lose their optimality in the multiprocessor case. Besides, the rate Monotonic (RM) and the Deadline Monotonic (DM) use a static priority and have many preemptions that may cause an over energy consumption. The table shows I a comparison of different schedulers. The G- EDF has proved to be optimal and has reasonable runtime overhead, this is why we chose it.

Several experiments of energy management [15]-[17] and real-time scheduling [18] are conducted. However, despite those energy-efficient architectures, few tools are available to test them. The simulator has to ensure the respect of the deadlines, an optimal throughput, and an efficient use of the resources [8]. As those simulators are legion, a very interesting open-source tool we came across was the STORM simulator. We have conducted a comparison of different relevant WSN simulators. We have classified as shown in figure 1 into energy-oriented or not simulator. A cross-level simulator with energy features is more suitable for WSN as it supports several abstraction levels combining high performance and scalability. That's why, we have chosen to validate our work with STORM [6].

\section{SYSTEM MODEL}

We propose a realistic power consumption model for WSN by incorporating the characteristics of a typical low power mote processor(the Mica2 platform) [19]. Measurements were conducted to determine the improvement in network lifetime based on the datasheet values of the ATMEGA128L. However, the model can be easily ported to other sensor nodes. The overall system consists of a large number of nodes distributed over a wide geographic area. Thus, the Fig. 2, shows the different internal interactions between the application layer and the hardware layer of every sensor through the intermediate layer that manages the resources. In our case, it provides the facilities to manage the CPU activity.

The first phase is to assign tasks to the nodes through the XML file. The inputs of the model are the data related to the parameters of time, number of tasks, the type of the processor, etc. The tasks are scheduled with the G-EDF policy. We consider $\mathrm{n}$ independent, periodic real-time tasks T1 ...Tn allocated to $\mathrm{m}$ identical processors $\mathrm{P} 1$...Pm with possible migration of tasks between the processors. In our case, a processor is the equivalent of one node. The objective is that all the tasks check their deadlines during the simulation. We assume that the overhead of a preemption is zero.

Then, the next phase aims to adapt the choice of the energy strategy according to the needs of the application. 
DVFS exploits the CMOS property that a linear reduction in the supply voltage results in a cubic reduction in the power consumption at the expense of a linear slow down in the processor frequency. DVFS schemes exploit this relationship to provide variable operating voltages and corresponding frequencies for the processor. There is a trade-off between the required execution time and the consumed power. So when the frequency is increasing, we have shorter execution and higher power consumption. However, when it is decreasing the execution time is longer and the power consumption is lower. The local approach consists of applying the DVFS to each processor. The overhead of changing DVFS settings are assumed to be negligible. To address this issue, only 3 values of supply voltage and corresponding operating frequency are selected as illustrated in Table II. To simplify the discussion, we consider that the voltage and the frequency are always adjusted together. When switching the voltage, we assume that the overhead associated with the scheduling of voltage is negligible.

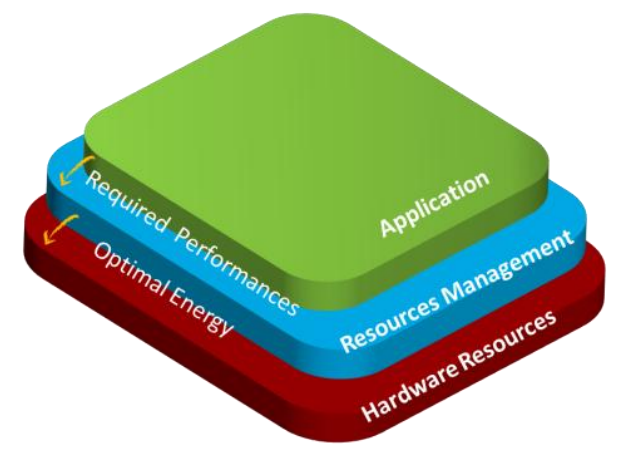

Fig. 2. Typical Interactions among the Layers of the WSN power manager.

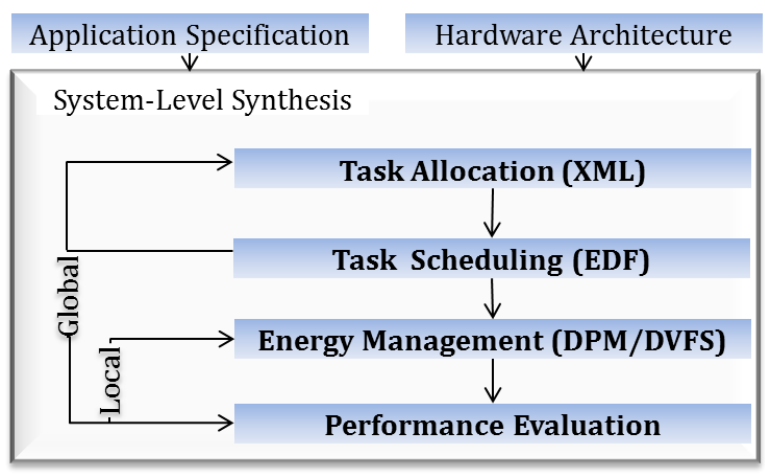

Fig. 3. Overview of the WSN energy model.

To get additional energy saving feature, we apply lowpower states during idle intervals. It is implemented in order to turn off tasks not running since they are consuming an important amount of energy by putting them in a low power consumption mode. The DPM policy used is different from the traditional WSN DPM policies by considering the penalties of transition modes and by an intelligent management of the energy consumption. Therefore, if a processor remains inactive for a significant period of time, it will automatically switch to a "standby" state during idle time. If this period of time is equal to the sum of the transition delays between these two states which allows us to avoid that the Wake up time reaches a high level. Thus, this online DPM will prolong the time spent in low power state. The extraction of idle times is done online and is based on the prediction of these intervals. This strategy includes the management of the idle time after the scheduling and is not involved in the scheduling of priorities. Finally, to evaluate and validate the results we will use the STORM simulator that considers the requirements of tasks, the characteristics and execution conditions of hardware compo- nents and the scheduling rules. Depending on the scheduling policy and the resources described in a XML file, it runs every task over a specified time interval [6]. The results of the simulation are a set of diagrams analyzing the behavior of the system (tasks, processors, timing, performances, etc) and mainly the energy consumption. Therefore, the behaviors of hardware components can be accurately captured, which is the basis of energy consumption estimation. Each state is associated with a current consumption. The simulator is easy and modular thanks to its XML configuration file.

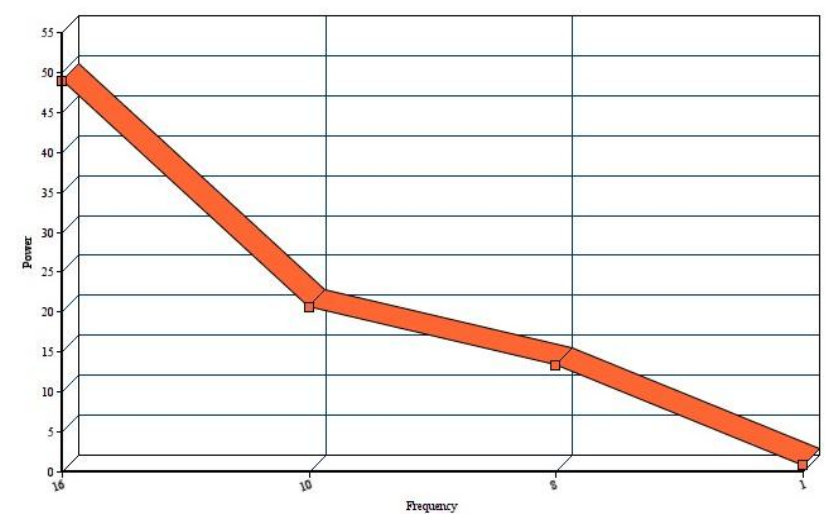

Fig. 4. Power consumption gain with DVFS.

\section{Evaluation of THE Power Model}

To evaluate optimal and autonomous power manager for sensor networks we have chosen the simulation. So, we have changed the network size (number of nodes) to study its impact on the whole system. We note that when increasing the number of tasks and keeping the same number of node that the rate of CPU utilization increases jointly up to $100 \%$. This is due to the fact that excessive request of the CPU had caused an overload.

While applying the DVFS, we found out that the power consumption is very likely larger at $16 \mathrm{MHz}$ than at $8 \mathrm{MHz}$. As the frequency will be reduced, the execution time will be increased and the idle time will be decreased. We have noted also that corollary to the scaling of the operating frequency by a factor $\alpha$, the worst calculation time (WCET) required by a task is multiplied by a factor $1 / \alpha$, whereas the desired period (and delay) remain unchanged. The proposed scheme is generic in the sense that it can work with other global scheduling algorithms as well rather than the EDF that is used. Our assessments indicate a gain is obtained using a DVFS algorithm in order to increase the autonomy of the mote. When the CPU frequency is equal to $\mathrm{fmax}=16 \mathrm{MHz}$ without DVFS, the frequency is lowered down to $8 \mathrm{MHz}$ with the proposed DVFS technique. Also, we have noticed that applying the scheduling when taking into account the current energy level and both of the power consumption and the priorities of the tasks (done through the EDF scheduler) had 
enabled graceful degradation. The average power consumption for both cases are $48.9 \mathrm{~W}(5.5 \mathrm{~V})$ and $20.6 \mathrm{~W}$ $(3.3 \mathrm{~V})$, respectively, which represent a $50 \%$ reduction in the total system energy.

$\mathrm{W}(3.3 \mathrm{~V})$, respectively, which represent a $50 \%$ reduction in the total system energy.

The Fig. 5 illustrates the experiment results obtained with the DPM strategy. The time is represented in units of 50 seconds(X-axis). Each task is represented by a different color.
We have noticed that it is not desirable to keep nodes inactive for too long because it can impact the network Qualityof-Service. When we had applied the DPM policy, major improvement had been seen such as the elimination of both dynamic and static power dissipation. Besides, the delay's transition had been set up to avoid the potential impact of missing the execution of any interesting task. Moreover, the transition between the different power configurations showed an extra energy and latency costs.

TABLE II: VOLTAGE/F R E QUENCY COUPLES SUPPORTED BY ATM EGA 128

\begin{tabular}{lccccc}
\hline \hline Level & 1 & 2 & 3 & Group 2 & Group $c$ \\
\hline Frequency(MHz) & 16 & 8 & 1 & Channel 2 & Channel $c$ \\
Voltage(V) & 5.5 & 3 & 2.7 & Channel 3 & Channel 1 \\
\hline \hline
\end{tabular}

TABLE III: COMPARISON OF GLOBAL AND LOCAL DVFS/DPM OUTPUT

\begin{tabular}{l||l||l}
\hline \multicolumn{1}{c||}{} & Global & Local \\
\hline \hline \multirow{2}{*}{ DPM } & Define a wide sleep state & Provide the necessary energy for each task \\
\cline { 2 - 3 } & \multicolumn{1}{c}{ Limitations: Mode-switching in CPU causes additional energy and latency penalty } \\
\cline { 2 - 3 } & Advantages: Easier to achieve & Advantages: Scalability \\
\cline { 2 - 3 } & Limitations: Difficult to model the idle interval & Limitations: Transition delay \\
\hline \hline \multirow{2}{*}{ DVFS } & A single global (F, V) to the whole network & Assign to each component's its (F, V) separately \\
\hline \hline & \multicolumn{2}{|c}{ Limitations: Lack of means to reduce static power consumption. } \\
\hline \hline & Advantages: Frequent, easy, cheap & Limitations: complexity \\
\hline \hline
\end{tabular}

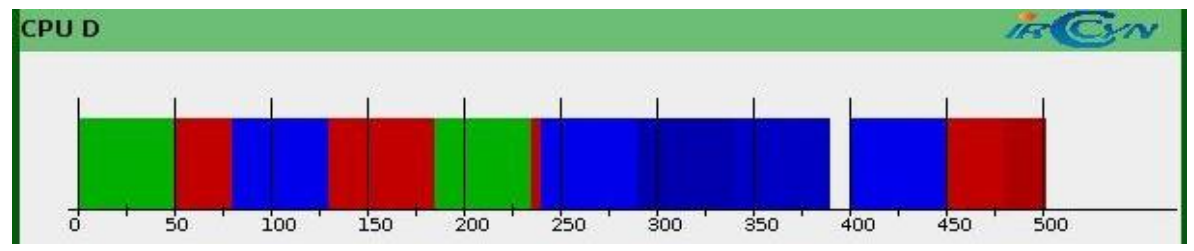

Fig. 5. EDF-DPM experiment results.

However, we have noticed that the costs of transition between modes but also the time spent by the CPU in each mode had a significant impact on the total consumption of energy of a sensor node.

As table III shows we have compared the output of the proposed energy model that combines using global and local DVFS/DPM techniques. Implementation and simulation have both shown that power-aware scheduling reduces the energy consumption at all levels of the network and that the network can adapt the scheduling locally to changing demand.

During the simulation, we have changed the network size (number of nodes) to study its impact on the whole system. As a result, we have noted that the more the number of processors increases, the more the processing time and the makespan(i.e. the date of completion of the last task scheduled) are reduced. However, it creates an additional energy costs by rising the slack time. The simulation demonstrates also how scaling further the frequencies can lead to more energy saving at the global level. The decision toward the length of an upcoming idle period in the DPM algorithm is not trivial.

\section{CONCLUSION}

To boost the power and the efficiency of the WSN, we have implemented an autonomous power management model that integrates the effects related to the hardware and that has been validated by the the STORM simulator. It checks whether the task execution should be carried out or not, depending on the available energy and the current time by exploiting dynamic voltage/frequency scaling (DVFS) and dynamic power man- agement (DPM) capabilities of the processor.

This model is formed by the various following modules: task allocation, task scheduling, energy management, and finally performance evaluation. We relied on a significant application example, and in our case it is the detection of forest fires in WSN. We have proven that, when applying an optimal combi- nation of the DVFS strategy (active state) and then DPM(idle state), the energy is not only locally minimized but also it decreases at a global level. Indeed, mixing both approaches has brought additional energy saving when deployed with a scheduling policy (G-EDF) since increasing the idle period without reducing the voltage or frequency will not necessarily bring an energy consumption gain. This power manager has a great impact on saving the energy reaching up to $50 \%$ of energy gain. We also, tried to extend STORM simulation tool and to enhance its features by including this model and some power management techniques that are not supported yet. 


\section{REFERENCES}

[1] J. A. Khan, H. K. Qureshi, and A. Iqbal, "Energy management in wireless sensor networks: A survey," Computers and Electrical Engineering, vol. 41, pp. 159-176, 2015.

[2] L. Angrisani, F. Bonavolonta, G. d'Alessandro, and M. D'Arco, "Inductive power transmission for wireless sensor networks supply," in Proc. IEEE Workshop on Environmental Energy and Structural Monitoring Systems(EESMS), 2014, pp. 1-5.

[3] I. Dietrich and F. Dressler, "On the lifetime of wireless sensor networks," ACM Transactions on Sensor Networks (TOSN), vol. 5, no. 1, p. 5, 2009.

[4] R. Cheour, F. Derbel, O. Kanoun, and M. Abid, "Wireless sensor networks with power management for low energy consumption," in Proc. of the2013 IEEE International Multi-Conference on Systems, Signals \& Devices (SSD), 2013, pp. 1-6.

[5] J. Wu and S. Yang, "Energy-efficient node scheduling models in sensor networks with adjustable ranges," International Journal of Foundations of Computer Science, vol. 16, no. 1, pp. 3-17, 2005.

[6] IRCCYN. (2016). Pre'sentation de storm. [Online]. Available: http://storm.rts- software.org/doku.php?id=start=

[7] B. Musznicki, and P. Zwierzykowski, "Survey of simulators for wireless sensor networks," Int J Grid Dist Comput, vol. 5, no. 3, pp. 23-50, 2012.

[8] R. Che'our, M. Jmal, A. Lay-Ekuakille, F. Derbel, O. Kanoun, and M. Abid, "Choice of efficient simulator tool for wireless sensor networks," in Proc. International Workshop on Measurements and Networking Proceedings, 2013.

[9] R. Khajuria, and S. Gupta, "Energy optimization and lifetime enhancement techniques in wireless sensor networks: A survey," in Proc. the 2015 IEEE International Conference on Computing, Communication \& Automation (ICCCA), 2015, pp. 396-402.

[10] K. Iniewski, C. Siu, S. Kilambi, S. Khan, B. Crowley, P. Mercier, and C. Schlegel, "Ultra-low power circuit and system design trade-offs for smart sensor network applications," in Proc. 2005 IEEE International Conference on Information and Communications Technology, 2005, pp. 309-321.

[11] J. Amaro, R. Cortesao, J. Landeck, and F. J. Ferreira, "Harvested power wireless sensor network solution for disaggregated current estimation in large buildings," IEEE Trans. Instrum. Meas., vol. 64, no. 7, pp. 1847-1857, 2015.

[12] M. A. Hannan, S. Mutashar, S. A. Samad, and A. Hussain, "Energy har- vesting for the implantable biomedical devices: issues and challenges," Biomed. Eng. Online, vol. 13, no. 1, p. 79, 2014.

[13] V. T. Hoang, N. Julien, and P. Berruet, "Increasing the autonomy of wireless sensor node by effective use of both dpm and dvfs methods," in Proc. IEEE Faible Tension Faible Consommation, 2013, pp. 1-4,

[14] M. Che'ramy, P.-E. Hladik, and A.-M. De'planche, "Simso: A simulation tool to evaluate real-time multiprocessor scheduling algorithms," in Proc. the 2014 the 5th International Workshop on Analysis Tools and Methodologies for Embedded and Real-time Systems, 2014, pp. 6-9..

[15] B. Marques and M. Ricardo, "Energy-efficient node selection in application-driven wsn," Wireless Networks, pp. 1-30, 2016.

[16] T. Rault, A. Bouabdallah, and Y. Challal, "Energy efficiency in wireless sensor networks: a top-down survey," Computer Networks, vol. 67, pp. 104-122, 2014.

[17] J. A. Khan, H. K. Qureshi, and A. Iqbal, "Energy management in wireless sensor networks: A survey," Computers and Electrical Engineering, 2014.
[18] D. Harkut, M. Ali, and P. Lohiya, "Scheduling task of wireless sensor network using earliest deadline first algorithm," International Journal of Scientific Research in Computer Science and Engineering, vol. 2, no. 02 , pp. 1-6, 2014

[19] D. Crossbow. (2015). Micazcrossbow datasheet. [Online]. Available: www.eol.ucar.edu/isf/facilities /isa/internal/ CrossBow/DataSheets/mica2.pdf

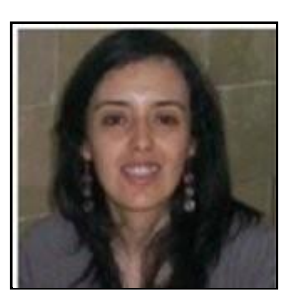

Rym Chéour received the engineering and the M.S. degrees from the National School of Engineers of Sfax, Tunisia in 2008 and 2009, respectively. She is currently working toward the Ph.D. degree in Department of Computer Science at Engineering National School of Sfax(ENIS), University of Sfax, Tunisia. Her research activities are conducted within CES Laboratory. They include Real Time schedul- ing, power management and wireless sensor net- works.

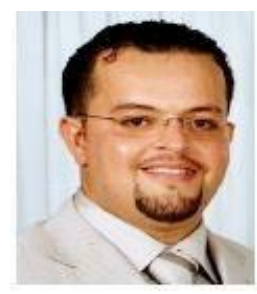

Mohamed Wassim JMAL is a Ph.D student at the National Engineering School of Sfax, Tunisia since 2008. His research activity is conducted within CES Laboratory. He has received the Engineering degree in Electrical Engineering, from the National Engineering School of Sfax in 2005 and the Mas- ter degree in automatic and industrial informat- ics, from the same Engineering School, in 2007. His current research interests are in the field of wireless sensor networks (WSN) and the embedded systems. They are focused on the implemen-tation of wireless sensor networks applications in reconfigurable system (http://www.ceslab.org/fr/perso.php?id=61). He has several publications in many conferences and Journals. He is working now as Assistant in Higher Institute of Applied Science and Technology of Gafsa, Tunisia. Mohamed Wassim JMAL served in national and international conference organization: IDT, ICM, TWESD, SensorNets

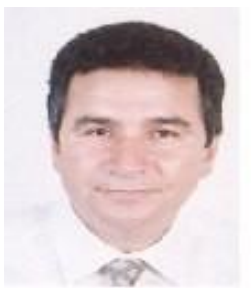

Mohamed Abid is working now as a professor at the Engineering National School of Sfax (ENIS), University of Sfax, Tunisia. He received the $\mathrm{Ph}$. D. degree from the National Institute of Applied Sciences, Toulouse (France) in 1989 and the "thèse $d$ état" degree from the National School of Engineering of Tu- nis(Tunisia) in 2000 in the area of computer en- gineering and microelectronics. His current research interests include are hardware software codesign, Sys- tem on Chip, reconfigurable system, and embedded system, etc. He has also been investigating the design and implementation issues of FPGA embedded systems. He is founding member and Head of the research laboratory, "Computer Embedded System" CES-ENIS, since 2006 (http:// www. ceslab.org). He was Founding member of System on Chip at Computer, Electronic and Smart engineering system Laboratory at ENIS 2001-2005 